\title{
Destress and do not suppress: targeting adrenergic signaling in tumor immunosuppression
}

\author{
Ignacio Iñigo-Marco ${ }^{1,2,3}$ and Marta M. Alonso $0^{1,2,3}$ \\ 'Navarra's Health Research Institute (IDISNA) Pamplona, Spain. ²Program in Solid Tumors, Foundation for Applied Medical Research, Pamplona, Spain. ${ }^{3}$ Department of Pediatrics, University Hospital of \\ Navarra, Pamplona, Spain.
}

\begin{abstract}
Tumor-induced immunosuppression is a common obstacle for cancer treatment. Adrenergic signaling triggered by chronic stress participates in the creation of an immunosuppressive microenvironment by promoting myeloid-derived suppressor cell (MDSC) proliferation and activation. In this issue of the $J C l$, Mohammadpour et al. elegantly delve into the mechanisms underlying MDSC contribution to tumor development. They used in vitro and in vivo mouse models to demonstrate that chronic stress results in MDSC accumulation, survival, and immune-inhibitory activity. Of therapeutic relevance, the authors showed that propranolol, a commonly prescribed $\beta$-blocker, can reduce MDSC immunosuppression and enhance the effect of other cancer therapies.
\end{abstract}

\section{Chronic adrenergic stress} impacts cancer progression

Chronic adrenergic stress has a deleterious effect on the immune system that impacts cancer progression, metastasis, and drug resistance $(1,2)$. Supporting this notion, previous studies showed that activating adrenergic receptors (ARs), which are broadly expressed in innate and adaptive immune cells and predominantly $\beta 2-\mathrm{AR}$, inhibits effector immune cells and promotes immunosuppressive cell activity (2). For example, $\beta 2$-AR activation leads to metabolic deprivation and decreased secretion of IFN- $\gamma$ by $\mathrm{CD}^{+} \mathrm{T}$ cells (3), impaired dendritic cell (DC) maturation (4), and increased myeloid-derived suppressor cell (MDSC) recruitment (5). In fact, many conventional anticancer therapies, as well as new immunotherapies, fail because the immune system is unable to fight the tumor. Tumors usually take advantage of immune system control mechanisms to create an appropriate microenvironment that favors cell survival and tumor growth (6). Tumors thrive in the presence of immunosuppressive cells and signals that inhibit apoptosis, promote angiogenesis, and prevent the recruitment of cytotoxic cells.

\section{Bridging stress and immunosuppression}

In this issue of the JCI, Mohammadpour et al. (7) go a step further to bridge stress and immunosuppression, dissecting the functional link between adrenergic stress and MDSCs in tumor development. The authors used orthotopic breast cancer mouse models and $\beta 2-\mathrm{AR}^{-/}$knockout mice, to demonstrate that stress caused by housing mice in subthermal conditions $\left(22^{\circ} \mathrm{C}\right)$ has a protumorigenic effect. They also showed adrenergic regulation of MDSCs as an effective strategy to restrain tumor growth and support other anticancer therapies (Figure 1) (7).

MDSCs are a heterogeneous group of immature myeloid cells. Given the appropriate signaling, these cells are able to differentiate into granulocytes, macrophages and dendritic cells. Due to their

- Related Article: p. 5537

Conflict of interest: The authors have declared that no conflict of interest exists.

Copyright: ( 2019 , American Society for Clinical Investigation.

Reference information: J Clin Invest. 2019;129(12):5086-5088. https://doi.org/10.1172/JCI133115.

immunosuppressive effects on the tumor microenvironment (TME) and their ability to promote tumor growth and dissemination, MDSCs are attracting much attention in cancer research. Aside from their direct immunosuppressive effects, they also indirectly promote tumorigenesis by acting upon other immune cells involved in tumor immunity; MDSCs secrete antiinflammatory cytokines such as IL-10 and TGF- $\beta$, affect NK function, attract Tregs, and suppress cytotoxic cells, among other functions.

Mohammadpour et al. thoroughly studied the role of MDSCs in a murine breast cancer model. They concluded that upon adrenergic stimulation, MDSCs activate and proliferate, leading to an overall protumoral microenvironment. Unlike MDSCs from $\beta 2-\mathrm{AR}^{-/-}$mice, MDSCs from WT mice presented several oncogenic features and exhibited increased expression of $\beta 2$-AR and tumoral markers, such as Arg-I, PDL-1, and BCL-2, among others (7).

Mohammadpour and colleagues revealed that MDSC protumoral changes depend on adrenergic-induced STAT3 phosphorylation (7). This finding seems reasonable since STAT3 is a transcription factor known to promote MDSC proliferation and survival while restraining their differentiation. Notably, STAT3 inhibition is harmless to normal cells. However, inhibiting STAT3 can trigger MDSCs to differentiate into tumor-associated macrophages (TAMs), which are myeloid cells that also play a preferentially immunosuppressive role in tumors (8). Therefore, inhibiting STAT3 would inhibit MDSC proliferation, but also increase the number of TAMs, which could have their own detrimental effects. If the overactivation of STAT3 found in tumors promotes MDSC proliferation but STAT3 inhibition induces MDSC differentiation into TAMs, what is less damaging: MDSC or TAM proliferation? Clinically targeting STAT3 to modulate MDSCs is an appealing strategy to 


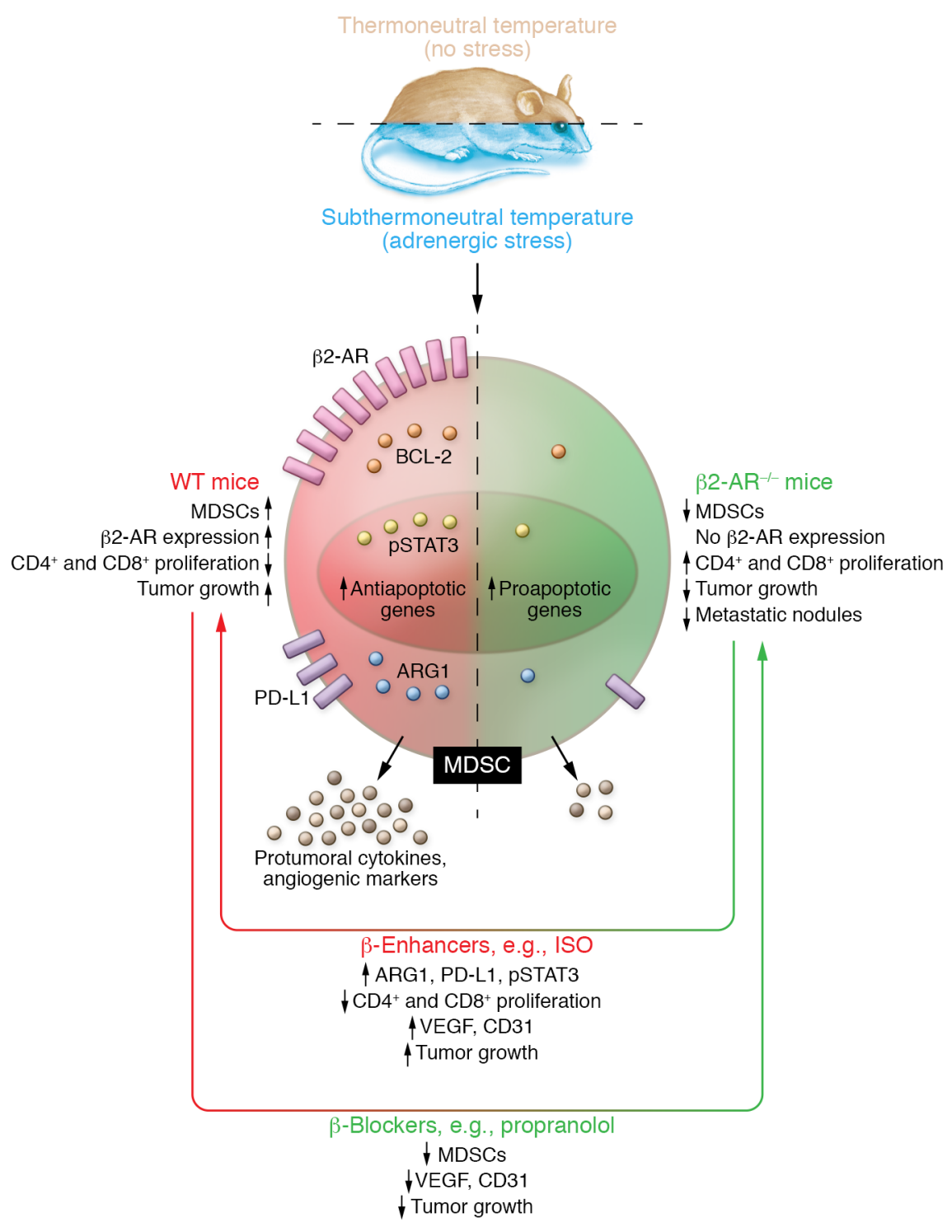

restrict immunosuppression in the TME; but if considered as a treatment option, studies should carefully check for opposing effects (9).

Mohammadpour and authors also showed that adrenergic-induced changes occur in MDSCs in both the TME and spleen (7). These changes in the spleen are remarkable since MDSCs sometimes behave differently in peripheral lymphoid organs as they do in the TME (10). MDSCs in peripheral lymphoid organs are mostly polymorphonuclear MDSCs (PMN-MDSCs), which depend on ROS-mediated immunosuppression and need antigen-specific interaction with $\mathrm{T}$ cells (11). In contrast, monocytic MDSCs (M-MDSCs), which are more abundant in the TME than PMN-MDSCs, have a more potent suppressive effect and rapidly differentiate
Figure 1. Schematic representation of adrenergic stress upon MDSC physiology. Subthermoneutral housing induces adrenergic $\beta 2$-AR-mediated stress. Adrenergic stress alters MDSC physiology and enhances MDSC protumoral-immunosuppressive signaling. MDSCs from $\beta 2-\mathrm{AR}^{-1-}$ mice fail to respond to adrenergic stress, which attenuates the immunosuppressive phenotype. $\beta$-enhancers, such as isoproterenol, enhance protumoral responses while the $\beta$-blocker propranolol alleviates protumoral phenotype in WT mice.

of cells, including immune cells, in which $\beta 2-\mathrm{AR}$ is the most highly expressed adrenergic receptor (15). Chronic adrenergic stress acts upon most of these immune cell types, compromising their function and leading to a poor patient outcome (1). Mohammadpour et al. confirm increased tumor growth under adrenergic stress. They showed that stress-promoting conditions increased MDSC proliferation and activation, as well as protumoral cytokine levels in plasma and tumor. Conversely, none of these effects are observed in $\beta 2-\mathrm{AR}^{-/-}$mice, which present a better outcome in terms of tumor growth, protumoral cytokine levels, and lung metastasis (7).

$\beta$-blockers in oncologic patients In their paper, Mohammadpour et al. not only confirm the immunosuppressive effect of MDSCs on tumor development, but also link this detrimental effect with stress-induced adrenergic signaling, which is common in oncologic patients. More importantly, the authors efficiently targeted and reverted the immunosuppressive phenotype using $\beta$-blocking drugs (7). $\beta$-blockers, such as propranolol, have treated other pathologies such as hypertension, essential tremor, anxiety, and migraines for decades. Propranolol has minor to no side effects and translational application would be much quicker and less expensive than new drug development. Notably, oncologic patients already taking $\beta$-blockers for other pathologies may have better outcomes (16). Although only one prospective study to date has addressed this finding, the results are encouraging. A fraction of melanoma patients taking propranolol showed a significantly lower rate of cancer recurrence than those not taking the medication (17). Nevertheless, clinical trials specifically designed to evaluate the safety and effectiveness of propranolol and other 
$\beta$-blockers in oncologic patients are necessary to elucidate the possible therapeutic value of $\beta$-blockers in a clinical context, which in turn will probably depend on many factors, such as the tumor type, disease stage, and patient age. Regardless, this study sheds light on tumor immunosuppressive mechanisms and reveals propranolol and other $\beta$-blockers as potential therapeutic tools in cancer treatment, and supports the idea of using $\beta$-blockers in those cases in which immunosuppression is an obstacle to common cancer therapies.

Mohammadpour et al. focused here on breast cancer, but the idea of translating these findings to other types of tumors in which treatment options are scarce and immunosuppression is a barrier to effective therapies, such as gliomas, is certainly exciting. Gliomas are primary brain tumors with a meager median survival and no curative therapies. Due to the highly immunosuppressed microenvironment of gliomas (reviewed in refs. 18 and 19), it would be truly interesting to see whether combining propranolol with different immunotherapy approaches could result in a curative therapy.

In closing, the work by Mohammadpour et al. provides a tangible link between chronic stress and immunosuppression, with implications in cancer pathogenesis and treatment (7). Moreover, this work challenges us to consider the stresses that accompany our daily routine and to take life with a grain of salt.

\section{Acknowledgments}

This work was supported by the Instituto de Salud Carlos III y los Fondos Feder Europeos (PI13/125 and PI16/0066 to MMA), Foundation Caja Navarra and Foundation LA CAIXA (to MMA), Foundation "El sueño de Vicky," Association Pablo Ugarte-Fuerza Julen (to MMA), and a DOD Team Science Award (CA160525 to MMA). This work was also supported by the European Research Council under the European Union's Horizon 2020 research and innovation programme, grant agreement 817884; ViroPedTher (to MMA).

Address correspondence to: Marta M. Alonso, Department of Pediatrics, Clínica Universidad de Navarra, CIMA Building, Avd. Pio XII, 55 Pamplona, Spain. Phone: 34.948194700.1017; Email: mmalonso@ unav.es.

1. Eng JW, Kokolus KM, Reed CB, Hylander BL, Ma WW, Repasky EA. A nervous tumor microenvironment: the impact of adrenergic stress on cancer cells, immunosuppression, and immunother apeutic response. Cancer Immunol Immunother. 2014;63(11):1115-1128.

2. Qiao G, Chen M, Bucsek MJ, Repasky EA, Hylander BL. Adrenergic signaling: a targetable checkpoint limiting development of the antitumor immune response. Front Immunol. 2018;9:164.

3. Estrada LD, Ağaç D, Farrar JD. Sympathetic neural signaling via the $\beta 2$-adrenergic receptor suppresses T-cell receptor-mediated human and mouse CD8(+) T-cell effector function. Eur J Immunol. 2016;46(8):1948-1958.

4. Mohammadpour H, O’Neil R, Qiu J, McCarthy PL, Repasky EA, Cao X. Blockade of host $\beta 2$ adrenergic receptor enhances graft-versustumor effect through modulating APCs. J Immunol. 2018;200(7):2479-2488.

5. Schmidt D, Peterlik D, Reber SO, Lechner A, Männel DN. Induction of suppressor cells and increased tumor growth following chronic psychosocial stress in male mice. PLoS One. 2016;11(7):e0159059.

6. Hui L, Chen Y. Tumor microenvironment: Sanctuary of the devil. Cancer Lett. 2015;368(1):7-13.

7. Mohammadpour $\mathrm{H}$, et al. $\beta 2$ adrenergic receptor-mediated signaling regulates the immunosuppressive potential of myeloidderived suppressor cells. J Clin Invest.
2019;129(12):5537-5552.

8. Kumar V, et al. CD45 phosphatase inhibits STAT3 transcription factor activity in myeloid cells and promotes tumor-associated macrophage differentiation. Immunity. 2016;44(2):303-315.

9. Su YL, Banerjee S, White SV, Kortylewski M. STAT3 in tumor-associated myeloid cells: multitasking to disrupt immunity. Int J Mol Sci. 2018;19(6):E1803.

10. Kumar V, Patel S, Tcyganov E, Gabrilovich DI. The nature of myeloid-derived suppressor cells in the tumor microenvironment. Trends Immunol. 2016;37(3):208-220.

11. Nagaraj S, Schrum AG, Cho HI, Celis E, Gabrilovich DI. Mechanism of T cell tolerance induced by myeloid-derived suppressor cells. J Immunol. 2010;184(6):3106-3116.

12. Haverkamp JM, et al. Myeloid-derived suppressor activity is mediated by monocytic lineages maintained by continuous inhibition of extrinsic and intrinsic death pathways. Immunity. 2014;41(6):947-959.

13. Weinstein LI, Revuelta A, Pando RH. Catecholamines and acetylcholine are key regulators of the interaction between microbes and the immune system. Ann N Y Acad Sci. 2015;1351:39-51.

14. Magnon C, et al. Autonomic nerve development contributes to prostate cancer progression. Science. 2013;341(6142):1236361.

15. Lorton D, Bellinger DL. Molecular mechanisms underlying $\beta$-adrenergic receptor-mediated crosstalk between sympathetic neurons and immune cells. Int JMol Sci. 2015;16(3):5635-5665.

16. Kokolus KM, et al. Beta blocker use correlates with better overall survival in metastatic melanoma patients and improves the efficacy of immunotherapies in mice. Oncoimmunology. 2018;7(3):e1405205.

17. De Giorgi V, et al. Propranolol for off-label treatment of patients with melanoma: results from a cohort study. JAMA Oncol. 2018;4(2):e172908.

18. Tomaszewski W, Sanchez-Perez L, Gajewski TF, Sampson JH. Brain tumor microenvironment and host state: implications for immunotherapy. Clin Cancer Res. 2019;25(14):4202-4210.

19. Binder DC, Davis AA, Wainwright DA. Immunotherapy for cancer in the central nervous system: Current and future directions. Oncoimmunology. 2016;5(2):e1082027. 